\title{
P53 expression correlates with poorer survival and augments the negative prognostic effect of MYC rearrangement, expression or concurrent MYC/BCL2 expression in diffuse large B-cell lymphoma
}

Xuan J Wang ${ }^{1}$, L Jeffrey Medeiros ${ }^{2}$, Carlos E Bueso-Ramos ${ }^{2}$, Guilin Tang ${ }^{2}$, Sa Wang ${ }^{2}$, Yasuhiro Oki ${ }^{3}$, Parth Desai ${ }^{2}$, Joseph D Khoury ${ }^{2}$, Roberto N Miranda ${ }^{2}$, Zhenya Tang ${ }^{2}$, Nishitha Reddy ${ }^{4}$ and Shaoying $\mathrm{Li}^{2}$

${ }^{1}$ Pathology, Vanderbilt University School of Medicine, Nashville, TN, USA; ${ }^{2}$ Department of Hematopathology, The University of Texas, MD Anderson Cancer Center, Houston, TX, USA; ${ }^{3}$ Department of Lymphoma \& Myeloma, The University of Texas, MD Anderson Cancer Center, Houston, TX, USA and ${ }^{4}$ Hematology/

Oncology, Vanderbilt University School of Medicine, Nashville, TN, USA

In patients with diffuse large B-cell lymphoma, MYC rearrangement (MYC-R), MYC expression, or concurrent expression of MYC and BCL2 is associated with a poorer prognosis. P53 expression also has been shown to confer inferior survival in diffuse large B-cell lymphoma patients, but less is known about the role of P53 expression in those with MYC-R, MYC expression (MYC+), or MYC\&BCL2 co-expression (MYC+/BCL2+). We studied P53 expression in 201 patients with untreated de novo diffuse large B-cell lymphoma. Sixty-seven (33\%) cases were P53 positive, 56 (28\%) had MYC-R (including 17 MYC/BCL2 double hit lymphoma), 86 (45\%) were MYC +/BCL2+, and 47 (24\%) were positive for both MYC and P53. Compared with patients with P53 negative lymphoma, the $\mathrm{P} 53$ positive group had a poorer overall survival $(P=0.004)$. In patients with lymphoma harboring $M Y C-R, M Y C$ expression or MYC+/BCL2+, P53 expression was associated with a significantly worse overall survival $(P<0.0001$, $P=0.01$, and $P=0.035$, respectively). Patients with lymphoma showing concurrent P53 expression and $M Y C$-R had a worse prognosis compared with patients with either P53 expression or $M Y C$ - $R$ alone $(\boldsymbol{P}<0.0001)$. Similarly, $P 53$ enhanced the negative prognostic effect of MYC expression in DLBCL patients. In addition, among patients with lymphoma with concurrent MYC and P53 expression, MYC and BCL2 or BCL2 \& P53 expression, those patients with tumors with MYC and P53 expression had the worst overall survival $(P=0.005)$, regardless of $B C L 2$ expression status. Multivariate analysis demonstrated that both $M Y C-R$ and P53 expression were independent prognostic factors in this patient cohort. In conclusion, our data suggest that P53 expression and MYC -R or MYC expression have an additive negative prognostic effect in diffuse large B-cell lymphoma patients. Assessment of P53 expression adds additional prognostic information in de novo diffuse large B-cell lymphoma patients, especially in subgroups with MYC-R, MYC expression and MYC and BCL2 double expression.

Modern Pathology (2017) 30, 194-203; doi:10.1038/modpathol.2016.178; published online 14 October 2016

Diffuse large B-cell lymphoma is the most common type of non-Hodgkin lymphoma worldwide. ${ }^{1}$ Cyclophosphamide, hydroxydaunorubicin, vincristine, and

Correspondence: Dr S Li, MD, Department of Hematopathology, The University of Texas, MD Anderson Cancer Center, 1515 Holcombe Blvd, Unit 72, Houston, TX 77030, USA.

E-mail: sli6@mdanderson.org

Received 31 May 2016; revised 30 August 2016; accepted 31 August 2016; published online 14 October 2016 prednisone (CHOP) has been the backbone of standard therapy for over four decades. The addition of rituximab to CHOP improved the 5-year overall survival, however, $\sim 40 \%$ of patients show primary refractory or disease relapse and have a poor prognosis. As the category of diffuse large B-cell lymphoma is highly heterogeneous and patients have variable outcomes, risk stratification of diffuse large B-cell lymphoma patients is an area of intense interest. The most commonly used prognostic 
predictors for diffuse large B-cell lymphoma patients are the International Prognostic Index (IPI) ${ }^{2,3}$ and the cell-of-origin classification (germinal center B-cell vs activated B-cell like). ${ }^{4}$ However, these tools do not completely account for the underlying tumor biology within each risk group. Therefore, additional biomarkers are needed to guide prognostic assessment and identify highest-risk patients for novel treatment approaches.

$M Y C$ has an important role in lymphomagenesis and has been well studied in diffuse large B-cell lymphoma. $M Y C$ is a transcription factor that is involved in cell cycle regulation, cell metabolism, mitochondrial biogenesis, nucleic-acid synthesis, and apoptosis. MYC translocation can upregulate many genes with growth-promoting effects resulting in exuberant cell proliferation. In addition to its proproliferative effect, studies also have shown that MYC upregulation is pro-apoptotic, limiting cell proliferation. ${ }^{5-8}$ MYC is rearranged, usually as a result of chromosomal translocation, in a number of different types of B-cell lymphoma including 10$15 \%$ of diffuse large B-cell lymphoma. ${ }^{9}$ In a subset of cases of diffuse large B-cell lymphoma with $M Y C$ rearrangement (MYC-R), BCL2 translocation also can occur, which results in $M Y C / B C L 2$ double-hit lymphoma. Presumably, overexpression of the anti-apoptotic protein BCL2 together with MYC expression leads to uncontrolled cell proliferation. Previous studies have shown that patients with diffuse large B-cell lymphoma with $M Y C$-R or $M Y C / B C L 2$ double hit have clinically aggressive disease and a poorer prognosis. ${ }^{6,10-21}$

Recent studies using immunohistochemical analysis have identified a subset of diffuse large B-cell lymphoma cases that express MYC and BCL2, known as double-positive lymphoma or double expresser lymphoma. Dual expression of MYC and BCL2 (MYC+/BCL2+) has been shown to be associated with a worse prognosis in patients with de novo diffuse large B-cell lymphoma treated with $\mathrm{R}$ CHOP. ${ }^{22,23}$ However, MYC/BCL2 double hit lymphoma and double-positive lymphoma are not concordant; double-positive lymphoma is much more common $(20-30 \%$ of diffuse large B-cell lymphoma) than double hit lymphoma $(\sim 10 \%$ of diffuse large B-cell lymphoma) and not all cases of double hit lymphoma express both MYC and BCL2.

TP53 is one of the most important molecular markers in cancer including diffuse large B-cell lymphoma. TP53 is a well-known tumor suppressor gene that is involved in many cell functions including cell cycle arrest, DNA repair, and activation of the pro-apoptotic machinery if cell repair mechanisms fail. Therefore, loss of normal P53 activity is related to lymphomagenesis and mediates tumor resistance to chemotherapy. TP53 is commonly mutated across different cancer types. In patients with diffuse large B-cell lymphoma, 20\% of cases are mutated and most TP53 mutations are thought to disrupt protein function and lead to disease progression. ${ }^{24,25}$ Previous studies have shown that TP53 mutation correlates with overexpression of P53 as shown by immunohistochemical assessment. Overexpression of P53 may be used as a surrogate of TP53 mutation and also is associated with poorer prognosis in patients with diffuse large B-cell lymphoma. ${ }^{24,26-30}$ However, little is known about the frequency of P53 expression, its prognostic role, and the significance of P53 expression in patients with diffuse large B-cell lymphoma with $M Y C$-R, MYC expression, $M Y C / B C L 2$ double hit lymphoma, or MYC and BCL2 coexpression. In this study we evaluated P53 expression and its prognostic impact in diffuse large B-cell lymphoma and specifically addressed P53 expression in cases of MYC/BCL2 double hit lymphoma, MYC +/BCL2+, and diffuse large B-cell lymphoma with $M Y C$-R or MYC expression.

\section{Materials and methods}

\section{Case Selection}

The archives of Vanderbilt and MD Anderson were searched for cases of de novo untreated DLBCL from 2010 to 2015 in which MYC status was tested by fluorescence in situ hybridization (FISH) analysis and/ or conventional cytogenetic analysis during routine diagnostic workup. The diagnosis of diffuse large B-cell lymphoma was based on the 2008 World Health Organization (WHO) criteria. $^{1}$ The following cases were excluded: (1) patients with a history of low-grade B-cell lymphoma; (2) post-transplant lymphoproliferative disorders or lymphomas arising in other immunocompromised situations; (3) primary mediastinal large B-cell lymphoma; (4) primary diffuse large B-cell lymphoma of the central nervous system; and (5) EBVpositive diffuse large B-cell lymphoma. A total of 201 cases with material available for further immunohistochemical analysis were included in this study. Corresponding medical records were reviewed to obtain clinical information, including history of lymphoma, number and sites of involvement, Ann Arbor stage, IPI, treatment regimens, response to therapy, and overall survival. Morphological, immunophenotypic, and cytogenetic data were reviewed to confirm the diagnosis. The study was approved by the institutional review boards.

\section{Immunophenotyping}

Immunohistochemical analysis was performed using formalin-fixed, paraffin-embedded tissue sections either at the time of diagnosis or retrospectively for this study. The panel of monoclonal antibodies included reagents specific for CD3 and CD20 (Ventana Medical Systems, Tucson, AZ, USA); CD5, CD10, BCL2, BCL6, and MUM1 (Leica Microsystems, Buffalo Grove, IL, USA); Ki-67 (MIB-1) (DAKO, Carpenteria, CA, USA); MYC (Epitomics, Burlingame, CA, USA) and P53 (Leica Biosystems, 
Newcastle, United Kingdom). Positivity for MYC, BCL2, and P53 expression was defined by using cutoffs of $\geq 40, \geq 50$, and $\geq 50 \%$ positive cells, respectively, as reported in previous studies. ${ }^{19,21,23,31,32}$

Flow cytometric immunophenotypic analysis was performed using 8-color flow cytometry. A FACScanto II or FACSCalibur cytometer (Becton-Dickinson Biosciences, San Jose, CA, USA) was used for analysis as described previously. ${ }^{31}$ Lymphocytes were gated using side scatter vs forward scatter, and CD45 expression vs side scatter. Antibody panels used included those specific for CD3, CD4, CD5, CD7, CD8, CD10, CD19, CD20, CD23, CD33, CD38, and immunoglobulin kappa and lambda light chains. All antibodies were obtained from BectonDickinson Biosciences.

\section{Conventional Cytogenetic Studies \& FISH}

Conventional G-band karyotype analysis was performed on 24 cases using cell suspensions of lymph node, extranodal tissue biopsy specimens, or bone marrow aspirate specimens. The karyotypes were reported according to the 2013 International System for Human Cytogenetic Nomenclature. ${ }^{33}$

FISH analysis was performed on all cases using a LSI MYC dual-color break-apart probe, and on most cases using LSI IGH@BCL2 dual-color, dual fusion probes, and LSI BCL6 dual color break-apart probe (Abbott Laboratories, Des Plaines, IL, USA). For formalin-fixed, paraffin-embedded tissue samples, FISH was performed on $4-\mu \mathrm{m}$ tissue sections and fixed onto slides as per the manufacturer's protocols. For bone marrow aspirate specimens, FISH was performed by using a freshly dropped slide from a harvested bone marrow aspirate specimen or a G-banded slide for metaphase mapping according to the manufacturer's instructions. The signals from 200 nuclei were analyzed. The cutoffs for considering a tumor sample rearranged for $M Y C, B C L 2$, and $B C L 6$ were different in bone marrow smears vs fixed, paraffin-embedded tissue sections. However, these cutoffs were low (all $\leq 5 \%$ ) and all cases in this study with $M Y C$ and/or BCL2 rearrangement had abnormal signals present in $>25 \%$ of all nuclei assessed. In earlier years, $M Y C, B C L 2$, and BCL6 FISH were performed together for a given newly diagnosed DLBCL case. However, our guidelines switched to only perform $M Y C$ FISH initially, and if positive then add on BCL2 and BCL6 in the recent 1-year interval. Therefore, BCL2 and BCL6 FISH were not performed in a small subset of cases.

\section{Statistical Analysis}

Overall survival was calculated by measuring the time elapsed from the date of diagnosis until the date of death or last patient follow-up. Patient overall survival was analyzed by the Kaplan-Meier method and compared using the log-rank test. Multivariate analysis was performed using Cox proportional hazards model. Statistical analysis was performed using SPSS 23 software. Differences in P53 positive $(\geq 50 \%)$ vs negative $(<50 \%)$ groups were analyzed using the Fisher exact test. A $P$-value of $<0.05$ was considered statistically significant.

\section{Results}

\section{Clinicopathologic Characteristics}

The study group included 132 men and 69 women with a median age of 64 years (range, 18-92). One hundred and twenty (60\%) patients were 60 years or older. One-third of patients had $\geq 2$ extranodal sites of disease, $25 \%$ had bone marrow, and 20\% had central nervous system involvement by lymphoma. The serum LDH level was increased in $57 \%$ of patients. Two-third of patients had stage III/IV disease and $56 \%$ belong to high-intermediate to high IPI categories. A total of 174 patients received immunochemotherapy: 106 received R-CHOP, 57 were treated with rituximab, etoposide, prednisone, vincristine, cyclophosphamide, and doxorubicin (REPOCH), and 11 received rituximab plus hyperfractionated cyclophosphamide, vincristine, doxorubicin, dexamethasone alternating with cytarabine and methotrexate (R-hyper-CVAD/Ara-C/MTX). Of these 174 patients, 17 received a stem cell transplant including 13 autologous and 4 allogeneic. The distribution of these 17 patients were not significantly different between the P53 $\geq 50 \%$ and $<50 \%$ groups (Table $1, P=0.27$ ), or between the $M Y C-\mathrm{R}+$ and $M Y C-\mathrm{R}$ - groups $(n=5$ vs $12, P=1.00)$. Therefore, they were included in further survival analysis. Twenty-seven patients received non-rituximab based treatment or lacked adequate clinical follow-up information and therefore are not included in further survival analysis.

P53 immunohistochemistry assessment showed nuclear expression with variable intensity. P53 expression ( $\geq 50 \%$ of cells) was observed in 67 of 201 (33\%) cases, including 23 with MYC-R (10 double hit lymphoma, 11 with neither BCL2 nor BCL6 rearrangement, and 2 with unknown BCL2 and BCL6 status) and 44 without $M Y C$-R (five with $B C L 2-\mathrm{R}, 9$ with $B C L 6$-R, and the remaining cases with neither BCL2-R nor BCL6-R or unknown status). Defined by immunohistochemistry, these $67 \mathrm{P} 53+$ cases included 36 MYC and BCL2 double-positive lymphoma (Figure 1), 11 with MYC but no BCL2 expression, 15 with BCL2 but no MYC expression, and 5 with neither MYC nor BCL2 expression. Compared with patients with diffuse large B-cell lymphoma without P53 expression, those with P53 expression had an increased frequency of elevated serum LDH level, central nervous system involvement, MYC expression, and MYC and BCL2 dual expression $(P<0.05$, Table 1). All other clinicopathologic features were 
Table 1 Clinicopathologic features of DLBCL according to P53 expression

\begin{tabular}{|c|c|c|}
\hline Features & $\begin{array}{c}P 53 \geq 50 \%(\mathrm{n}=67) \\
\% \text { (positive) } \\
\text { evaluated) }\end{array}$ & $\begin{array}{c}P 53<50 \%(\mathrm{n}=134) \\
\% \text { (positive/ } \\
\text { evaluated) }\end{array}$ \\
\hline $\begin{array}{l}\text { Age (years), } \\
\text { Median (range) }\end{array}$ & $66(22-87)$ & $63(18-92)$ \\
\hline Age $\geq 60$ (years) & $66(44 / 67)$ & $57(76 / 134)$ \\
\hline Male & $66(44 / 67)$ & $67(88 / 134)$ \\
\hline BM positive & $33(16 / 48)$ & $21(22 / 104)$ \\
\hline CNS positive* & $35(9 / 26)$ & $11(5 / 44)$ \\
\hline Extranodal sites $\geq 2$ & $37(25 / 67)$ & $37(50 / 134)$ \\
\hline $\begin{array}{l}\text { Elevated serum } \\
\text { LDH }^{*}\end{array}$ & $68(36 / 53)$ & $52(57 / 110)$ \\
\hline Stage III or IV & $72(42 / 58)$ & $68(80 / 125)$ \\
\hline $\begin{array}{l}\text { High-intermediate/ } \\
\text { high IPI }\end{array}$ & $61(34 / 56)$ & $54(64 / 118)$ \\
\hline \multicolumn{3}{|l|}{ Immunophenotype } \\
\hline CD10 & $56(37 / 66)$ & $56(73 / 131)$ \\
\hline BCL2 & $75(51 / 65)$ & $83(109 / 131)$ \\
\hline $\mathrm{MYC}^{*}$ & $70(47 / 67)$ & $47(59 / 125)$ \\
\hline $\begin{array}{l}\text { MYC/BCL2 } \\
\text { co-expression* }\end{array}$ & $55(36 / 65)$ & $40(50 / 125)$ \\
\hline $\begin{array}{l}\text { Ki67 } \geq 70 \% \\
\text { (range) }\end{array}$ & $83(40-100)$ & $78(5-100)$ \\
\hline $\begin{array}{l}\% \text { of GCB } \\
\text { subtype }\end{array}$ & $76(44 / 58)$ & $68(80 / 118)$ \\
\hline \multicolumn{3}{|l|}{ FISH } \\
\hline$M Y C-\mathrm{R}$ & $34(23 / 67)$ & $25(33 / 134)$ \\
\hline$B C L 2-\mathrm{R}$ & $24(14 / 59)$ & $27(32 / 115)$ \\
\hline DHL/THL & $17(10 / 59)$ & $11(13 / 115)$ \\
\hline \multicolumn{3}{|l|}{ Initial chemotherapy } \\
\hline R-CHOP & $59(33 / 56)$ & $62(73 / 118)$ \\
\hline R-EPOCH & $36(20 / 56)$ & $31(37 / 118)$ \\
\hline R-HCVAD & $5(3 / 56)$ & $7(8 / 118)$ \\
\hline With SCT & $5(3 / 56)$ & $12(14 / 118)$ \\
\hline $\begin{array}{l}\text { Median OS } \\
\text { (months)* }\end{array}$ & 40 & 110 \\
\hline
\end{tabular}

${ }^{*} P<0.05$. OS

similar between the P53+ and P53 - groups $(P>0.05$, Table 1).

Prognostic Significance of $M Y C$-R, BCL2-R, MYC/BCL2 Double Hit Lymphoma and Expression of MYC, BCL2 or Both MYC and BCL2 in Patients with Diffuse Large B-cell Lymphoma

All cases had MYC cytogenetic status confirmed by FISH at the time of diagnosis. Fifty-six of $201(28 \%)$ cases had $M Y C$-R, including 17 (8.5\%) MYC/BCL2 double hit lymphoma, 4 (2\%) MYC/BCL2/BCL6 triple hit lymphoma, 2 (1\%) MYC/BCL6 double hit lymphoma, $15(7.5 \%)$ MYC-R only, and $18(9 \%)$ cases with $M Y C$-R, but unknown BCL2 or/and BCL6 status. BCL2 and BCL6 status was assessed by FISH in 175 and 142 cases, respectively (see methods section). Forty-six of 175 (26\%) cases showed BCL2$\mathrm{R}$ and 33 of $142(23 \%)$ showed BCL6-R.

MYC expression was detected in 106 of $192(55 \%)$ cases and BCL2 in 160 of 196 (82\%); MYC and
BCL2 were co-expressed in 86 of 190 (45\%) cases. Similar to the results of previous studies in the literature, $M Y C$-R, $M Y C / B C L 2$ double hit lymphoma and MYC expression were each associated with a significantly worse overall survival $(P<0.05$, Figures 2a-C). Patients with MYC and BCL2 double expression also had a worse OS compared with nondouble expression patients with a borderline $P$-value $(P=0.067$, Figure 2d). In contrast, BCL2 rearrangement and BCL2 expression each did not correlate with OS ( $P>0.05$, Figures 2e and $\mathrm{f})$.

\section{Prognostic Significance of P53 Expression in Patients with Diffuse Large B-cell Lymphoma, Diffuse Large B-cell Lymphoma with MYC-R, MYC/BCL2 Double Hit Lymphoma, MYC Expression or MYC and BCL2 Double Expression}

P53 expression was present in 67 of 201 (33\%) diffuse large B-cell lymphoma patients. P53 expression was associated with a significantly poorer overall survival, with a median overall survival of 40 months in the P53+ group vs not reached in P53 - group (Figure 3a, $P=0.006)$. The prognostic significance of P53 expression was further analyzed in known aggressive subsets of diffuse large B-cell lymphoma patients. In 56 patients who had diffuse large B-cell lymphoma associated with $M Y C-\mathrm{R}$, P53 expression was associated with a significantly worse overall survival compared with those without P53 expression (median OS 7.4 vs 67 months, $P<0.0001$; Figure $3 \mathrm{~b}$ ). This effect was not affected by BCL2 status $(P=0.24$, Figure 3c). Analysis in the subgroup of patients with MYC/BCL2 double hit lymphoma showed a similar effect although the $P$-value was borderline, perhaps owing to the limited number of cases $(P=0.06$; Figure 3d). Similarly, in 106 patients with diffuse large B-cell lymphoma associated with MYC expression, P53 expression conferred a poorer OS $(P=0.015$, Figure 3e). In 86 patients with MYC/BCL2 double expression lymphoma, the 36 patients with tumors also positive for P53 expression had a shorter overall survival compared with 50 patients with double expression lymphoma but without P53 expression (20 vs 67 months, $P=0.035$; Figure $3 f$ ). The prognostic significance of P53 expression was further analyzed in patients with diffuse large B-cell lymphoma harbor $B C L 2-\mathrm{R}$ or BCL6-R. When all cases with BCL2-R or $B C L 6$ - R were included, P53 expression predicted a worse overall survival in patients with $B C L 2$-R tumors $(P=0.046)$ and a trend toward worse overall survival in patients with $B C L 6$-R tumors $(P=0.063)$, however, these effects were lost when cases with $M Y C$-R were excluded ( $P=0.50$ and $P=0.32$, respectively), suggesting those prognostic effects were actually related to the presence of $M Y C-R$. 

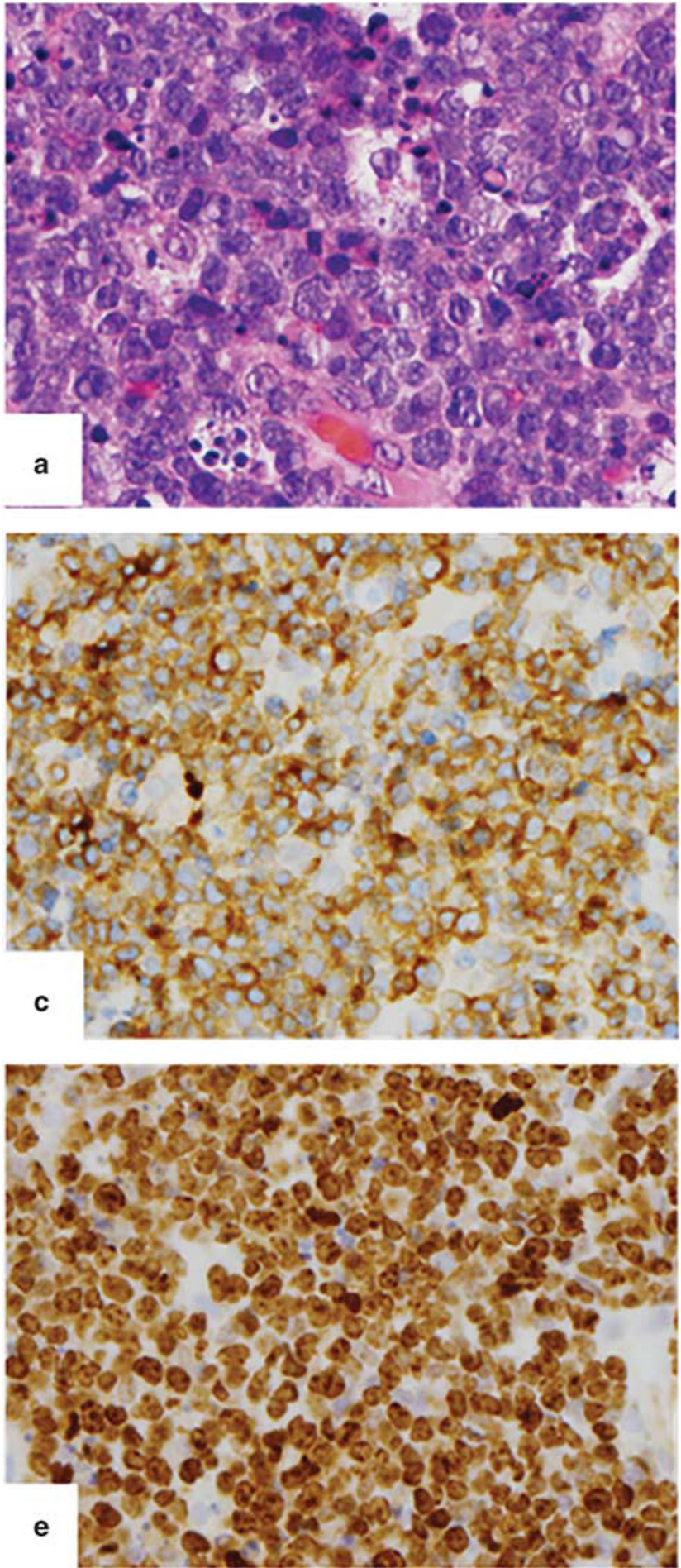
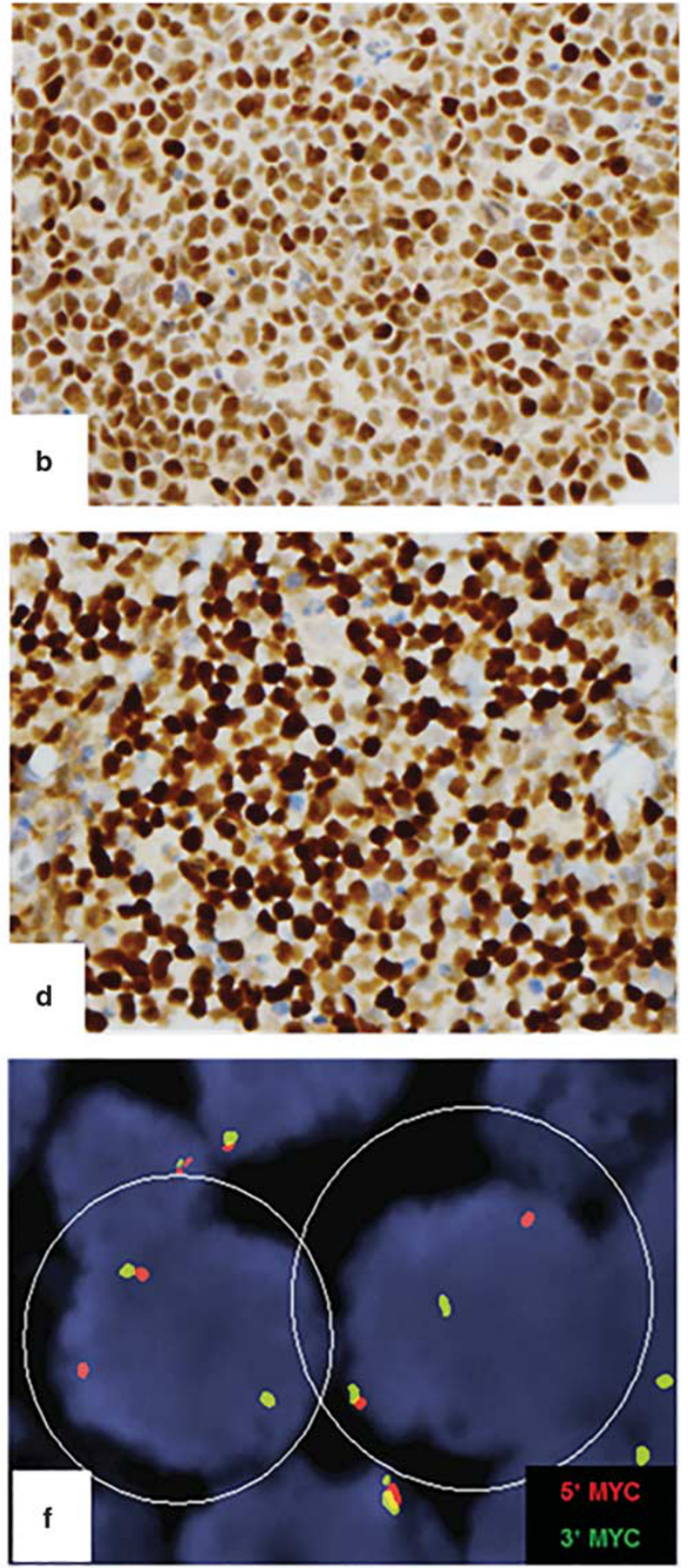

Figure 1 (a) A representative case of diffuse large B-cell lymphoma (a, h, and $\mathbf{e}, \times 600)$ with high MYC (b, × 400, 100\% positivity), BCL2 (c, $\times 400,90 \%$ positivity), P53 (d, ×400, 95\% positivity), and Ki67 (e, ×400, 100\%) expression by immunohistochemistry, and MYC rearrangement (f) by FISH.

P53 Augments the Negative Prognostic Effect of MYC-R or MYC Expression

MYC-R, MYC expression and P53 expression all have a negative prognostic effect in patients with diffuse large B-cell lymphoma, as shown above. In patients with diffuse large B-cell lymphoma characterized by both P53 expression and MYC-R (Figure 4a), the OS was significantly worse than for patients with diffuse large B-cell lymphoma associated with only $M Y C$-R or 


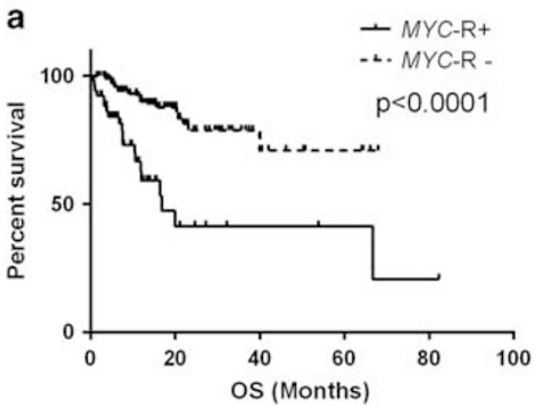

b
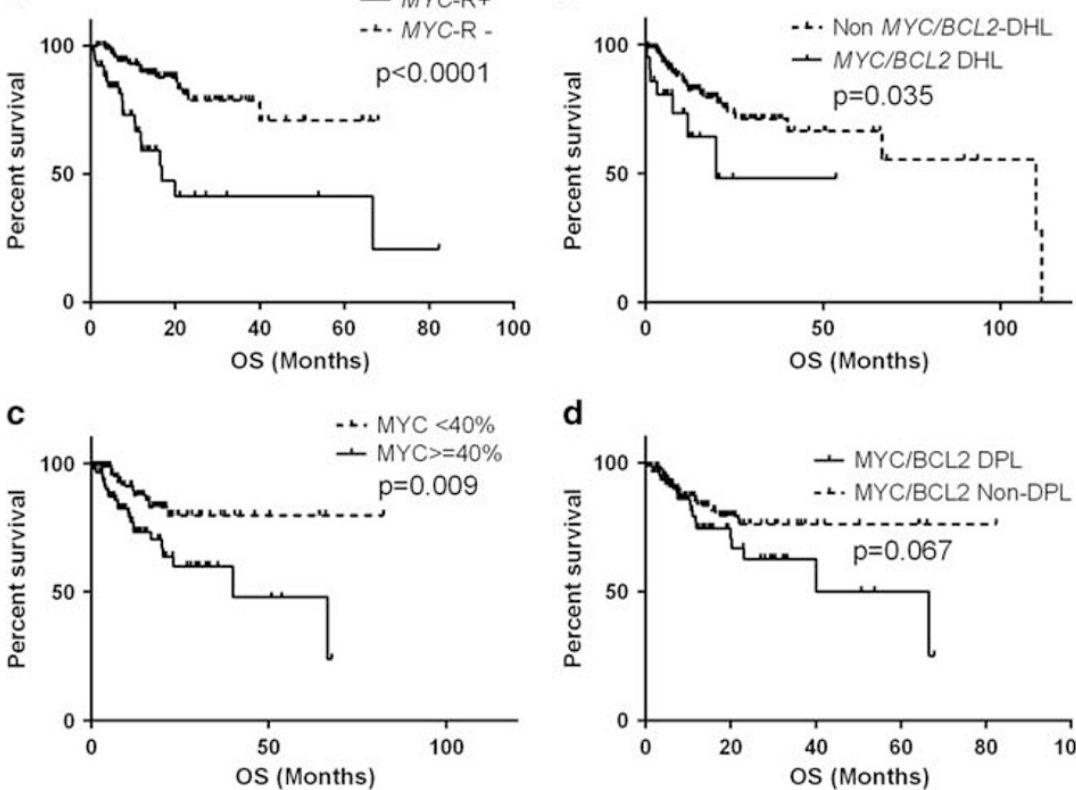

d
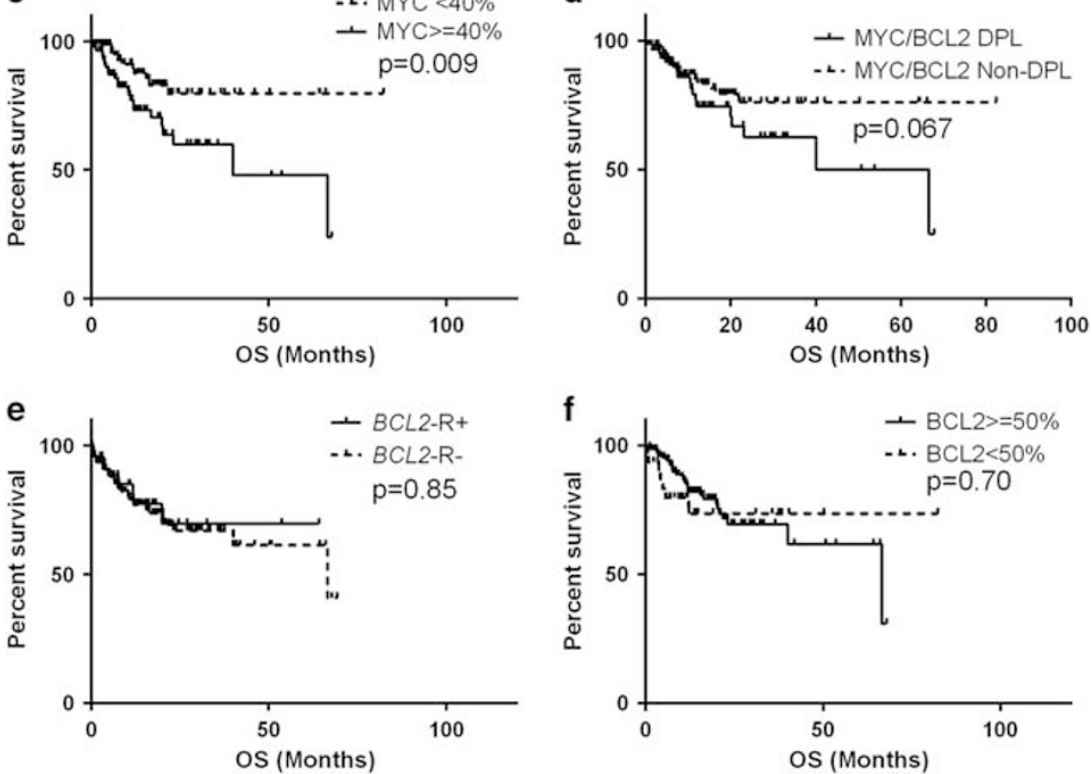

Figure 2 Overall survival by (a) MYC rearrangement (MYC-R); (b) MYC/BCL2 DHL; (c) MYC expression; (d) Concurrent MYC and BCL2 expression (DPL); (e) BCL2 rearrangement (BCL2-R); and (f) BCL2 expression.

P53 expression $(P<0.0001)$. P53 expression was not associated with a significant worse overall survival in patients with diffuse large B-cell lymphoma without $M Y C$-R. In patients with P53p-negative diffuse large B-cell lymphoma, cases with $M Y C$-R had a similar overall survival compared with those without $M Y C-\mathrm{R}$ (Figure 4a). These results suggest that P53 expression enhanced the negative prognostic effect of $M Y C$-R.

Patients with diffuse large B-cell lymphoma without MYC and P53 expression had the best overall survival (Figure 4b); those with only P53 or MYC expression had a worse overall survival; and patients with lymphoma showing both P53 and MYC expression had the poorest OS, significantly worse than patients with tumors with only P53 or MYC expression $(P<0.0001)$. These results suggest that $\mathrm{P} 53$ expression intensified the negative prognostic effect of MYC expression. In addition, MYC expression was associated with P53 expression: MYC expression was present in 47 of $67(70 \%)$ diffuse large B-cell lymphoma cases with P53 expression, significantly more common than that seen in cases that lacked of P53 expression (59 of 125; 47\%) $(P=0.002$, Table 1). Further analysis showed that patients with lymphoma positive for both MYC and P53 (with or without BCL2 expression) had significantly worse overall survival compared with patients with MYC/ BCL2 double-positive lymphoma without P53 expression as well as patients with tumor with BCL2 and P53 expression but no MYC expression $(P<0.05)$ (Figure 4c). All of these subsets had a worse overall survival than patients with diffuse large B-cell lymphoma without MYC, BCL2, and P53 expression $(P=0.005)$. These results further suggest an additive negative prognostic effect seen between P53 and MYC expression.

\section{Multivariate Survival Analysis}

The IPI is a well-established prognostic factor in patients with diffuse large B-cell lymphoma. Highintermediate/high IPI scores were associated with a worse overall survival in this patient cohort by univariate analysis $P<0.0001)$. MYC-R, MYC expression, P53 expression, and IPI were therefore entered in a multivariate analysis. The results showed that IPI, MYC-R, and P53 expression were independent prognostic factors in this patient cohort (Table 2). 

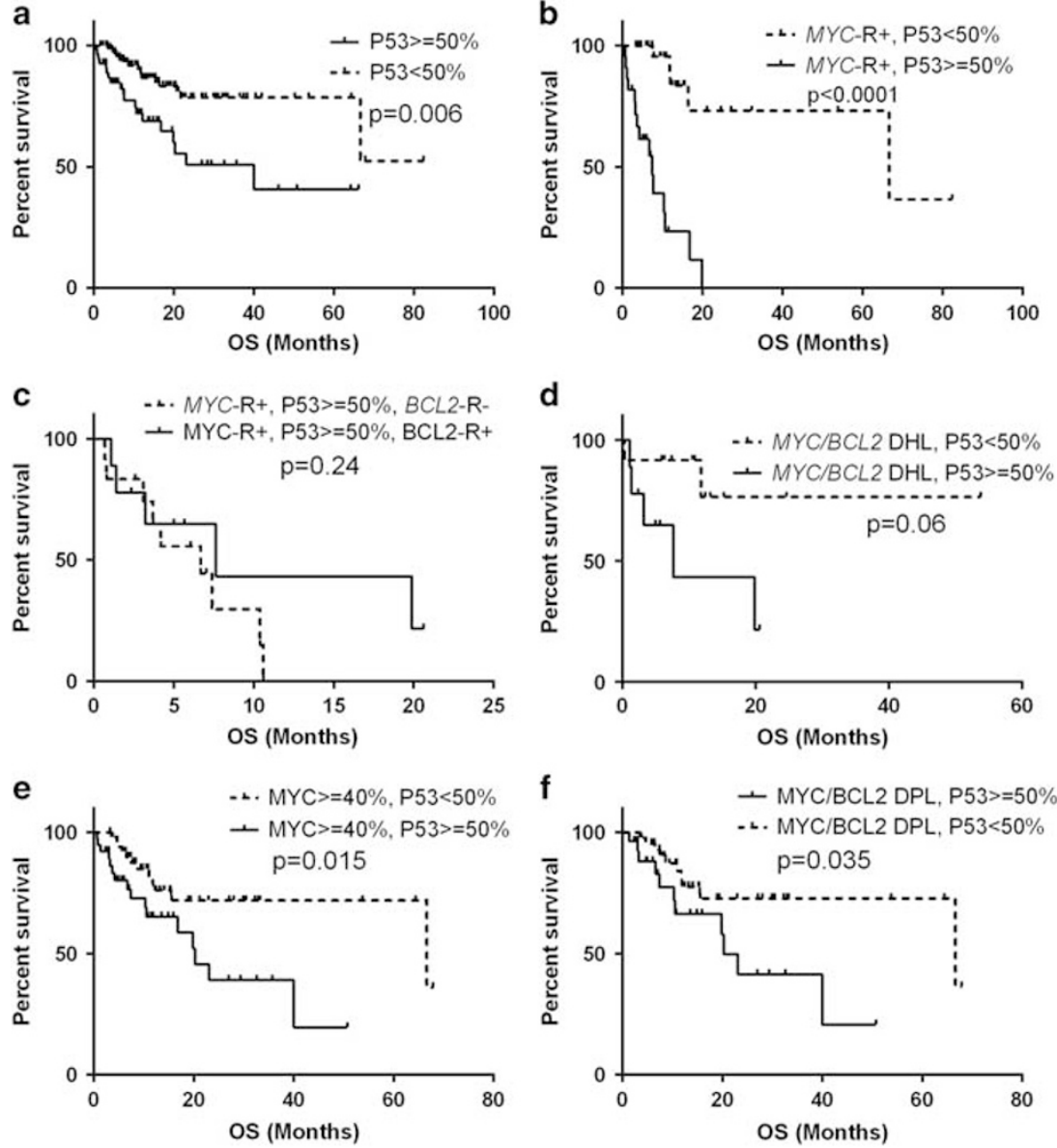

Figure 3 The effect of P53 expression on overall survival (OS) in patients with (a) diffuse large B-cell lymphoma; (b) diffuse large B-cell lymphoma with $M Y C$ rearrangement (MYC-R); (c) $M Y C$-R with or without BCL2-R; (d) MYC/BCL2 double hit lymphoma (DHL); (e) MYC expression, and (f) concurrent MYC and BCL2 expression (DPL).

a

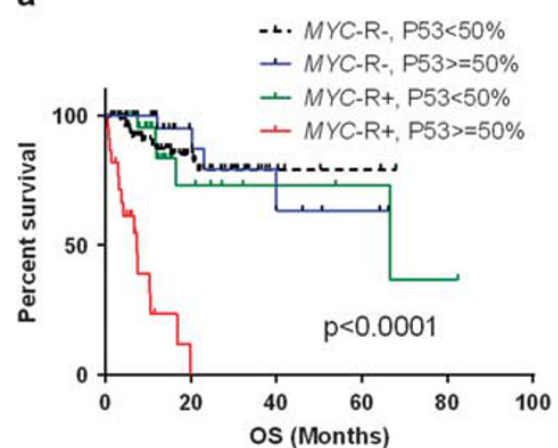

b

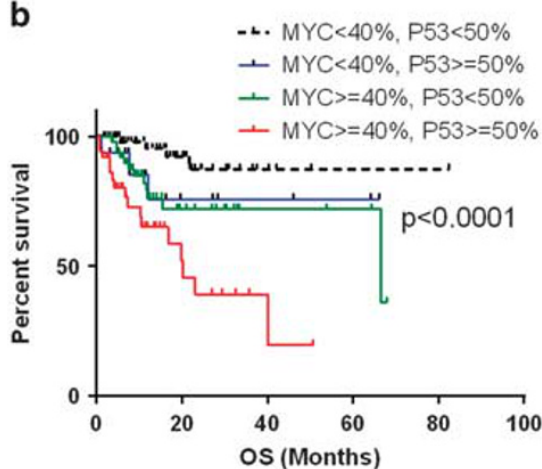

C $\quad-\mathrm{MYC}+/ \mathrm{P} 53+, \mathrm{BCL} 2>=50 \%$

$+\mathrm{MYC}+/ \mathrm{P} 53+, \mathrm{BCL} 2<50 \%$

$-\mathrm{MYC}+/ \mathrm{BCL} 2+, \mathrm{P} 53<50 \%$

$-\mathrm{BCL} 2+/ \mathrm{P} 53+, \mathrm{MYC}<40 \%$

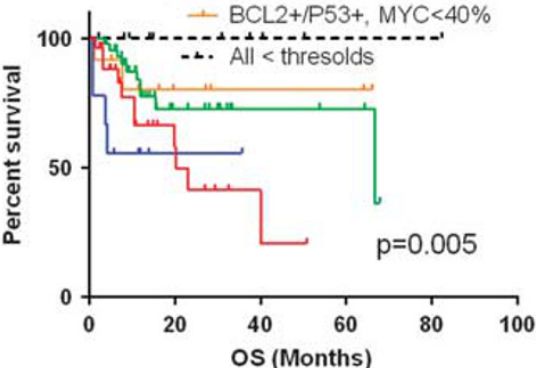

Figure 4 The additive negative effect of P53 expression and (a) MYC rearrangement (MYC-R); (b) MYC expression; and (c) concurrent MYC and BCL2 expression on overall survival.

\section{Discussion}

It is known that P53 overexpression is associated with a poor prognosis in patients with diffuse large B-cell lymphoma. However, information regarding the role of P53 expression in clinically aggressive subsets of patients with diffuse large B-cell lymphoma, such as tumors associated with $M Y C-\mathrm{R}$,
Table 2 Multivariate analysis of overall survival

\begin{tabular}{lccc}
\hline Features & HR & $95 \%$ CI & P-value \\
\hline MYC-R by FISH & 2.92 & $1.440-5.938$ & 0.003 \\
MYC expression & 1.49 & $0.652-3.383$ & 0.346 \\
P53 expression & 1.88 & $1.037-3.585$ & 0.038 \\
IPI (H/H-I vs L/L-I) & 6.25 & $2.176-17.925$ & 0.001 \\
\hline
\end{tabular}


MYC/BCL2 double hit lymphoma, MYC expression, or MYC/BCL2 double expression is limited.

It is known that P53 mutation occurs in $~ 20 \%$ of cases of diffuse large B-cell lymphoma and patients with mutated tumors have a poorer prognosis. ${ }^{24,29,34,35}$ The prognostic significance of P53 expression is controversial. Some studies have reported that P53 expression is a poor prognostic factor in diffuse large B-cell lymphoma, ${ }^{24,27}$ whereas others have shown no prognostic effect. ${ }^{36-38}$ Multiple factors likely contribute to this inconsistency: different patient cohorts with variable clinical features; diverse biological features such as molecular subtypes; differences in treatment regimens used; and different P53 cutoff levels employed. The prevalence of $\mathrm{P} 53$ expression in this patient cohort was $33 \%$ using a cutoff of $50 \%$ positive cells, similar to that of other studies in the literature in which 20$50 \%$ of cases of diffuse large B-cell lymphoma were reported to be positive. ${ }^{24,26,29,36,39,40}$ In this study, P53 expression was associated with a shorter overall survival in diffuse large B-cell lymphoma patients using any cutoff from 20 to $50 \%$ in a univariate analysis (results not shown). However, in multivariate analysis only a 50\% cutoff showed that overexpression of $\mathrm{P} 53$ is an independent poor prognostic factor in patients with diffuse large B-cell lymphoma treated with rituximab based chemotherapy. We also show that P53 expression is associated with a higher rate of central nervous system involvement, higher frequency of an elevated serum $\mathrm{LDH}$ level, and higher frequencies of MYC expression and MYC/BCL2 co-expression. Our results further confirm that P53 overexpression have a role in the prognosis of patients with DLBCL.

A number of reports in the literature have shown that $M Y C$-R, MYC expression, $M Y C / B C L 2$ double hit lymphoma and MYC/BCL2 double-positive lymphoma are associated with a poorer prognosis. In this study we extend these observations by showing that P53 expression is also a poor prognostic factor in patients with diffuse large B-cell lymphoma with $M Y C$-R, MYC expression, MYC/BCL2 double hit lymphoma, and MYC/BCL2 double expression lymphoma. These data suggest that assessment of P53 expression in diffuse large B-cell lymphoma adds additional prognostic value in patients with aggressive subsets of disease.

Our results also suggest an additive effect between P53 expression and MYC-R or MYC expression. A previous study by Xie et al. ${ }^{27}$ showed an enhanced negative effect of MYC and P53 expression in diffuse large B-cell lymphoma patients. In their study, 16 patients with concurrent P53 and MYC expression showed a worse overall survival compared with 19 patients with only P53 expression, 7 patients with only MYC expression, or those patients with lymphoma with neither P53 nor MYC expression. In the current analysis, patients with lymphoma with only MYC $(n=56)$ or P53 $(n=20)$ expression had a poorer overall survival than those without P53 and MYC expression, whereas those with both P53 and MYC expression $(n=47)$ showed the poorest survival, worse than P53 or MYC expression alone. In addition, we further analyzed the effect of P53 in MYC/BCL2 double-positive lymphoma and demonstrated that expression of P53 predict a significant shorter survival. We compared patients with P53 \& MYC, P53 \& BCL2, and those with MYC and BCL2 co-expression but no P53 expression, and showed that patients with P53 and MYC co-expression had the worst OS, regardless of BCL2 expression status. These data suggest that concurrent expression of P53 and MYC had an additive negative prognostic effect in diffuse large B-cell lymphoma patients. Our results further demonstrated that P53 expression and $M Y C$-R also have an additive negative prognostic effect in diffuse large B-cell lymphoma patients, which has not been reported previously.

The observation that $\mathrm{P} 53$ and $M Y C-\mathrm{R}$ or MYC expression have an additive negative prognostic effect in diffuse large B-cell lymphoma patients likely could be explained by what is currently known about these genes. TP53 is a tumor suppressor gene and is known as the 'guardian of the genome'. TP53 responds to a variety of signals that stress cells including hypoxia, DNA damage (any etiology), and hyperproliferative signals from oncogenes. MYC is an oncogene that can cause lymphoma by its prominent pro-proliferative activity when rearranged or overexpressed. When MYC-induced hyperproliferation occurs, normal P53 will induce cell cycle arrest at the G1 phase of the cell cycle, allowing cells to recover and further repair their damage, or to induce apoptosis to eliminate damaged cells that are not amenable to repair. These functions may prevent lymphoma development or facilitate favorable lymphoma treatment effect by causing lymphoma cell apoptosis during chemotherapy. However, when TP53 is mutated, these tumor suppression functions are lost. Mutated TP53 encodes a protein with a longer half-life than wildtype protein and therefore cells are positive for P53 when assessed by immunohistochemistry. As cell stress and DNA damage increase, lymphoma cells with P53 overexpression likely fail to undergo cell cycle arrest, DNA repair, or apoptosis. Rather, these cells continue to proliferate, allowing for additional oncogenic alterations, and therefore clinically more aggressive disease.

In conclusion, the results presented suggest that P53 expression is an independent prognostic factor in patients with untreated de novo diffuse large B-cell lymphoma and that P53 expression adds additional prognostic information in patients with diffuse large B-cell lymphoma associated with $M Y C$ R, MYC expression and MYC/BCL2 double expression. In addition, P53 expression and MYC appear to have an additive negative prognostic effect in diffuse large B-cell lymphoma patients, regardless of BCL2 status. These results suggest that immunohistochemical assessment for P53 in diffuse large B-cell 
lymphoma is a practical approach to further improve risk stratification of diffuse large B-cell lymphoma patients, especially when TP53 mutation status is unknown.

\section{Disclosure/conflict of interest}

The authors declare no conflict of interest.

\section{References}

1 Stein H, Chan JKC, Warnke RA, et al. Diffuse large B cell lymphoma, not otherwise specified. In: Swerdlow SH CE, Harris NL, Jaffe ES, et al. (eds). WHO Classification of Tumours of Haematopoietic and Lymphoid Tissues. 4th Edn. IARC: Lyon, 2008, pp 233-237.

2 A predictive model for aggressive non-Hodgkin's lymphoma. The International Non-Hodgkin's Lymphoma Prognostic Factors Project. N Engl J Med 1993;329:987-994.

3 Sehn LH, Berry B, Chhanabhai M, et al. The revised International Prognostic Index (R-IPI) is a better predictor of outcome than the standard IPI for patients with diffuse large B-cell lymphoma treated with R-CHOP. Blood 2007;109:1857-1861.

4 Alizadeh AA, Eisen MB, Davis RE, et al. Distinct types of diffuse large B-cell lymphoma identified by gene expression profiling. Nature 2000;403:503-511.

5 Klapproth K, Wirth T. Advances in the understanding of MYC-induced lymphomagenesis. $\mathrm{Br} \mathrm{J}$ Haematol 2010;149:484-497.

6 Li S, Lin P, Young KH, et al. MYC/BCL2 double-hit high-grade B-Cell lymphoma. Adv Anat Pathol 2013;20: 315-326.

7 Dang CV, O'Donnell KA, Zeller KI, et al. The c-Myc target gene network. Semin Cancer Biol 2006;16: 253-264.

8 Nilsson JA, Cleveland JL. Myc pathways provoking cell suicide and cancer. Oncogene 2003;22:9007-9021.

9 Slack GW, Gascoyne RD. MYC and aggressive B-cell lymphomas. Adv Anat Pathol 2011;18:219-228.

10 Aukema SM, Siebert R, Schuuring E, et al. Double-hit B-cell lymphomas. Blood 2011;117:2319-2331.

11 Li S, Lin P, Fayad LE, et al. B-cell lymphomas with MYC/8q24 rearrangements and IGH@BCL2/t(14;18) (q32;q21): an aggressive disease with heterogeneous histology, germinal center B-cell immunophenotype and poor outcome. Mod Pathol 2012;25:145-156.

12 Tomita N, Tokunaka M, Nakamura N, et al. Clinicopathological features of lymphoma/leukemia patients carrying both BCL2 and MYC translocations. Haematologica 2009;94:935-943.

13 Johnson NA, Savage KJ, Ludkovski O, et al. Lymphomas with concurrent BCL2 and MYC translocations: the critical factors associated with survival. Blood 2009;114:2273-2279.

14 Kluin PM HN, Stein H, Leoncini L. B-cell lymphoma, unclassifiable, with features intermediate between diffuse large B-cell lymphoma and Burkitt lymphoma. In: Swerdlow SH, Campo E, Harris NL, et al. (eds). WHO Classification of Tumours of Haematopoietic and Lymphoid Tissues. 4th edn. IARC: Lyon, 2008, pp 265-267.
$15 \mathrm{Li} \mathrm{S}$, Weiss VL, Wang XJ, et al. High-grade B-cell Lymphoma With MYC Rearrangement and Without BCL2 and BCL6 Rearrangements Is Associated With High P53 Expression and a Poor Prognosis. Am J Surg Pathol 2015;40:253-261.

16 Savage KJ, Johnson NA, Ben-Neriah S, et al. MYC gene rearrangements are associated with a poor prognosis in diffuse large B-cell lymphoma patients treated with R-CHOP chemotherapy. Blood 2009;114:3533-3537.

17 Snuderl M, Kolman OK, Chen YB, et al. B-cell lymphomas with concurrent IGH-BCL2 and MYC rearrangements are aggressive neoplasms with clinical and pathologic features distinct from Burkitt lymphoma and diffuse large B-cell lymphoma. Am J Surg Pathol 2010;34:327-340.

18 Valera A, Lopez-Guillermo A, Cardesa-Salzmann T, et al. MYC protein expression and genetic alterations have prognostic impact in patients with diffuse large B-cell lymphoma treated with immunochemotherapy. Haematologica 2013;98:1554-1562.

19 Wang XJ, Medeiros LJ, Lin P, et al. MYC cytogenetic status correlates with expression and has prognostic significance in patients with MYC/BCL2 protein double-positive diffuse large B-cell lymphoma. Am J Surg Pathol 2015;39:1250-1258.

20 Oki Y, Noorani M, Lin P, et al. Double hit lymphoma: the MD Anderson Cancer Center clinical experience. Br J Haematol 2014;166:891-901.

21 Li S, Saksena A, Desai P, et al. Prognostic impact of history of follicular lymphoma, induction regimen and stem cell transplant in patients with MYC/BCL2 double hit lymphoma. Oncotarget 2016;7:38122-38132.

22 Green TM, Young KH, Visco C, et al. Immunohistochemical double-hit score is a strong predictor of outcome in patients with diffuse large B-cell lymphoma treated with rituximab plus cyclophosphamide, Doxorubicin, vincristine, and prednisone. J Clin Oncol 2012;30:3460-3467.

23 Johnson NA, Slack GW, Savage KJ, et al. Concurrent expression of MYC and BCL2 in diffuse large B-cell lymphoma treated with rituximab plus cyclophosphamide, doxorubicin, vincristine, and prednisone. J Clin Oncol 2012;30:3452-3459.

24 Xu-Monette ZY, Wu L, Visco C, et al. Mutational profile and prognostic significance of TP53 in diffuse large B-cell lymphoma patients treated with R-CHOP: report from an International DLBCL Rituximab-CHOP Consortium Program Study. Blood 2012;120:3986-3996.

25 Xu-Monette ZY, Medeiros LJ, Li Y, et al. Dysfunction of the TP53 tumor suppressor gene in lymphoid malignancies. Blood 2012;119:3668-3683.

26 Chang CC, Liu YC, Cleveland RP, et al. Expression of c-Myc and p53 correlates with clinical outcome in diffuse large B-cell lymphomas. Am J Clin Pathol 2000;113:512-518.

27 Xie Y, Bulbul MA, Ji L, et al. p53 expression is a strong marker of inferior survival in de novo diffuse large B-cell lymphoma and may have enhanced negative effect with MYC coexpression: a single institutional clinicopathologic study. Am J Clin Pathol 2014;141: 593-604.

28 Gebauer N, Bernard V, Gebauer W, et al. TP53 mutations are frequent events in double-hit B-cell lymphomas with MYC and BCL2 but not MYC and BCL6 translocations. Leuk Lymphoma 2014;56:179-185.

29 Kerbauy FR, Colleoni GW, Saad ST, et al. Detection and possible prognostic relevance of p53 gene mutations in 
diffuse large B-cell lymphoma. An analysis of 51 cases and review of the literature. Leuk Lymphoma 2004;45: 2071-2078.

30 Koduru PR, Raju K, Vadmal V, et al. Correlation between mutation in P53, p53 expression, cytogenetics, histologic type, and survival in patients with B-cell non-Hodgkin's lymphoma. Blood 1997;90:4078-4091.

31 Li S, Seegmiller AC, Lin P, et al. B-cell lymphomas with concurrent MYC and BCL2 abnormalities other than translocations behave similarly to MYC/BCL2 double-hit lymphomas. Mod Pathol 2015;28:208-217.

32 Li S, Weiss VL, Wang XJ, et al. High-grade B-cell lymphoma with MYC rearrangement and without BCL2 and BCL6 rearrangements is associated with high P53 expression and a poor prognosis. Am J Surg Pathol 2016;40:253-261.

33 Shaffer LG M-JJ, Schmid M. An International System for Human Cytogenetic Nomenclature (2013). 1st edn. KargerBasel: Switzerland, 2012.

34 Young KH, Weisenburger DD, Dave BJ, et al. Mutations in the DNA-binding codons of TP53, which are associated with decreased expression of TRAIL receptor-2, predict for poor survival in diffuse large B-cell lymphoma. Blood 2007;110:4396-4405.

35 Ichikawa A, Kinoshita T, Watanabe T, et al. Mutations of the p53 gene as a prognostic factor in agg- ressive B-cell lymphoma. N Engl J Med 1997;337: $529-534$

36 Sohn SK, Jung JT, Kim DH, et al. Prognostic significance of bcl-2, bax, and p53 expression in diffuse large B-cell lymphoma. Am J Hematol 2003;73: 101-107.

37 Maartense E, Kramer MH, le Cessie S, et al. Lack of prognostic significance of BCL2 and p53 protein overexpression in elderly patients with diffuse large B-cell non-Hodgkin's lymphoma: results from a populationbased non-Hodgkin's lymphoma registry. Leuk Lymphoma 2004;45:101-107.

38 Rujirojindakul P, Aiempanakit K, Kayasut K, et al. No prognostic impact of p53 and P-glycoprotein expression in patients with diffuse large B-cell lymphoma. ISRN Oncol 2011;2011:670358.

39 Fiskvik I, Beiske K, Delabie J, et al. Combining MYC, BCL2 and TP53 gene and protein expression alterations improves risk stratification in diffuse large B-cell lymphoma. Leuk Lymphoma 2015;56: 1742-1749.

40 Paik JH, Jeon YK, Park SS, et al. Expression and prognostic implications of cell cycle regulatory molecules, p16, p21, p27, p14 and p53 in germinal centre and non-germinal centre B-like diffuse large B-cell lymphomas. Histopathology 2005;47:281-291. 